\title{
Power corrections in heavy-to-light decays at large recoil energy *
}

\author{
M. Diehl ${ }^{\mathrm{a}}$ \\ anstitut für Theoretische Physik E, RWTH Aachen, 52056 Aachen, Germany \\ I briefly present recent work on QCD power corrections in heavy-to-light meson decays, using an effective field \\ theory approach.
}

\section{INTRODUCTION}

The strong interaction physics in $B$ decays is characterized by the presence of several distance scales. The general idea of factorization in QCD is to separate the dynamics at short distances (which can be treated perturbatively) from the long-distance physics (encoded in hadronic matrix elements like decay constants or form factors). There are different techniques to achieve this separation. One is the detailed analysis of Feynman graphs, used for instance in [1] to establish QCD factorization for exclusive $B$ decays. Here I focus on an effective field theory approach, whose principal objects are operators and fields. This framework is very general and well suited to be applied to a wide class of processes.

As an explicit application I consider exclusive $B$ decays to light mesons (e.g. $B \rightarrow \pi \ell \nu$, $\left.B \rightarrow K^{*} \ell^{+} \ell^{-}\right)$in kinematics where the recoil energy of the light meson is large, $E \sim m_{b}$. In the limit $m_{b} \rightarrow \infty$ symmetry relations between the various form factors describing the $B \rightarrow \pi$ or $B \rightarrow K^{*}$ transition emerge [2], which greatly reduce hadronic uncertainties when analyzing these decays in the Standard Model and beyond. These relations receive corrections in inverse powers of $m_{b}$, which we have systematically studied in effective field theory [3]. They also receive corrections in $\alpha_{s}$ from the heavy quark decay vertex (which are part of the effective theory) and from hard interactions of the spectator quark in the $B$ meson (which have so far only been treated by direct calculation of the relevant Feynman graphs in QCD 跑).

\footnotetext{
*Talk given at ICHEP 2002, Amsterdam, July 2002. Based on work with A. Chapovsky, M. Beneke and Th. Feldmann [3]
}

\section{EFFECTIVE FIELD THEORY}

To set up the effective field theory appropriate for a given process one first identifies the relevant momentum regions in the corresponding Feynman graphs. One wants to keep in the theory only the nearly on-shell momentum modes that directly connect to the external hadrons. In the decays of our study (see Fig. 1) this is a heavy quark in the initial $B$ meson, a cluster of light quarks and gluons which move approximately collinear to each other and form the fast outgoing meson, and "ultrasoft" gluons in either the initial or final state. (At this point we exclude the hard spectator quark interactions mentioned above.) Modes with large momenta $k^{\mu} \sim m_{b}$ are "integrated out" from the theory: this is done in perturbation theory and provides the Wilson coefficients associated with the shaded blob in the figure. The same happens to "soft" gluons with momenta $k^{\mu} \sim \sqrt{m_{b} \Lambda_{\mathrm{QCD}}}$. The heavy quark and its interactions with ultrasoft gluons is described in conventional heavy-quark effective theory (HQET), and the dynamics of collinear and ultrasoft modes in the soft-collinear effective theory (SCET) pioneered by Bauer et al. [5].

For each momentum mode a separate field is introduced, e.g. $A_{c}^{\mu}(x)$ and $A_{u s}^{\mu}(x)$ for collinear and ultrasoft gluons. For these fields one then must find the Lagrangian and current operators which reproduce the results of QCD to a given accuracy in a power-counting parameter $\lambda$, in our case given by $\sqrt{\Lambda_{\mathrm{QCD}} / m_{b}}$. It proves useful to project out the large spinor components of fermion fields and to integrate out the small ones. In HQET this is the usual projection on $h_{v}(x)=$ $\frac{1}{2}(1+\psi) Q_{v}(x)$, where $Q_{v}(x)=e^{i m_{b}(v x)} Q(x)$ has 


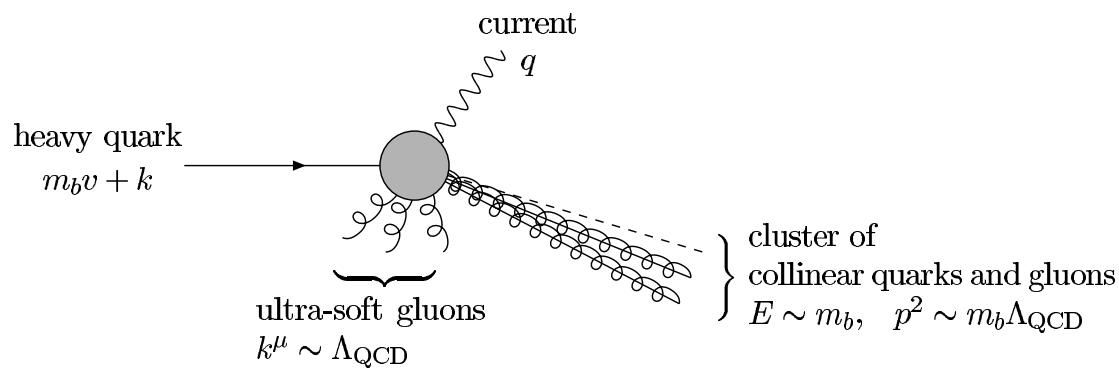

Figure 1. Heavy-quark decay into a cluster of collinear and ultrasoft particles.

the large phase of the full heavy quark field removed. For collinear quarks the large component is

$\xi(x)=\frac{1}{4} \not h_{-} \not h_{+} q(x)$,

where $n_{-}$and $n_{+}$are lightlike reference vectors, $n_{-}$giving the direction of the collinear particles and $n_{+}$the opposite direction. In contrast to [5] we do not split off the large phase of collinear fields in our approach [3]. For ultrasoft quarks all spinor components are of same size and hence kept in the theory.

To decide whether an operator is relevant to a given accuracy in $\lambda$ one determines power counting rules for momentum components (collinear momenta are large in the $n_{-}$direction and small in the others), for fields, their derivatives, etc. Finally, the effective theory "inherits" part of the gauge invariance of full QCD, and one has to define appropriate gauge transformations for the effective fields to implement this.

\subsection{The effective Lagrangian}

To obtain the effective Lagrangian for collinear and ultrasoft quarks and gluons one can start from the QCD quark Lagrangian $\mathcal{L}=\bar{q} i \not D q$, separate out the different momentum modes, and integrate out the small spinor components of the collinear quark field (using e.g. path integral methods). The result is

$\mathcal{L}_{c}=\bar{\xi}\left(i n_{-} D+i \not D_{\perp} W \frac{1}{i n_{+} \partial} W^{\dagger} i \not D_{\perp}\right) \frac{h_{+}}{2} \xi$ if one neglects ultrasoft quarks. Because of the inverse derivative $\left(n_{+} \partial\right)^{-1}$ the action is nonlocal. It is explicitly gauge invariant: the sum $A=A_{c}+A_{u s}$ of gluon fields appears in the covariant derivative $D=\partial-i g A$ and in the Wilson line

$W(x)=P \exp \left\{i g \int_{-\infty}^{0} d s n_{+} A\left(x+s n_{+}\right) \cdot\right\}$

One must still expand $\mathcal{L}_{c}$ in $\lambda$ : the components of $A_{c}^{\mu}$ and $A_{u s}^{\mu}$ have different scaling in $\lambda$, which requires a Taylor expansion of $D$ and $W$. Furthermore, $A_{u s}(x)$ varies more slowly in $x$ than collinear fields with their large-momentum modes, so that $A_{u s}(x)$ must be appropriately Taylor expanded in $x$.

To leading order in $\lambda$ ultrasoft quarks just appear in $\mathcal{L}_{u s}=\bar{q}_{u s} i D_{u s} q_{u s}$, but in higher orders they couple to collinear fields. The resulting Lagrangian including $O(\lambda)$ and $O\left(\lambda^{2}\right)$ corrections also contains ultrasoft Wilson lines $Z$, obtained from $W$ by setting $A_{c}=0$.

\subsection{The effective heavy-to-light current}

Before decaying via an electroweak current a heavy quark can radiate collinear gluons, which puts it off shell by an amount of order $m_{b}^{2}$. The corresponding diagrams have no place in the effective theory and must be summed into an effective vertex. This leads one to match the QCD heavy quark field onto an effective field, $Q \rightarrow e^{-i m_{b}(v x)} Q_{\text {eff }}\left(A_{c}, A_{u s}, h_{v}\right)$. By explicitly summing diagrams or by solving the Dirac equation for $Q_{\text {eff }}$ in an external field $A=A_{c}+A_{u s}$ 
one obtains $Q_{\text {eff }}$ as

$W Z^{\dagger} Q_{v}-\frac{1}{\mathcal{V}^{2}-1}\left(\mathcal{\psi} W Z^{\dagger}-W Z^{\dagger} \mathcal{Y u s}_{\mathrm{us}} \mathcal{Y}_{\mathrm{us}}\right) Q_{v}$

including corrections of order $\lambda$ and $\lambda^{2}$. Here $\mathcal{V}^{\mu}=v^{\mu}+i D^{\mu} / m_{b}$ is the reparametrization invariant "velocity" known from HQET.

Matching of the QCD light quark field onto an effective field, $q \rightarrow q_{\text {eff }}\left(A_{c}, A_{u s}, \xi\right)$, is readily performed in SCET. The complete QCD current is matched as $\bar{q} \Gamma Q \rightarrow e^{-i m_{b}(v x)} \bar{q}_{\mathrm{eff}} \Gamma Q_{\mathrm{eff}}$ and finally has to be Taylor expanded in $\lambda$ as in the case of the effective Lagrangian.

\section{POWER CORRECTIONS TO FORM FACTORS}

The symmetry relations for $E, m_{b} \rightarrow \infty$ between the transition form factors from $B$ to a light meson $L=\pi, \rho, K^{*}, \ldots$ receive corrections in powers of $\lambda=\sqrt{\Lambda_{\mathrm{QCD}} / m_{b}}$, which can be systematically described in the effective theory of Sec. 2 by matching the QCD matrix elements $\langle L|\bar{q} \Gamma Q| B\rangle$ onto $\left\langle L\left|\bar{q}_{\text {eff }} \Gamma Q_{\text {eff }}\right| B\right\rangle$. Projecting $\bar{q}_{\text {eff }}$ and $Q_{\text {eff }}$ on their large and small spinor components one finds that

1. at leading order in $\lambda$ only the large components are relevant. This gives 1 (2) independent form factors for the transition to a pseudoscalar (vector) meson [2]4].

2. the $O(\lambda)$ corrections involve the small components of either $\bar{q}_{\text {eff }}$ or $Q_{\text {eff }}$, and one remains with 2 (5) independent form factors.

3. the $O\left(\lambda^{2}\right)$ corrections come from the small components of both fields. Hence no symmetry relations are left to this accuracy, and one has 3 (7) independent form factors as in full QCD.

There is a caveat concerning the corrections at order $\lambda$. The corresponding operators in the effective theory involve different parton configurations in the light meson than the leading-order operators, for instance with an additional transversely polarized gluon. There may well be further dynamical suppression of their matrix elements, bringing them down from $O(\lambda)$ to $O\left(\lambda^{2}\right)$.
Whether this happens requires knowledge of the light meson wave functions which goes beyond the scope of SCET. We remark that these putative $O(\lambda)$ corrections affect the form factor relations used in the analysis of the forward-backward asymmetry in $B \rightarrow K^{*} \ell^{+} \ell^{-}$, see [6].

\section{SUMMARY}

We have performed a systematic analysis of power corrections to soft-collinear factorization in the effective field theory formalism. The interactions between collinear and ultrasoft quarks and gluons are described in SCET, whose effective Lagrangian was obtained including the power corrections of first and second order in $\lambda$. Heavyto-light meson decays at large recoil energy can be described by the effective actions of SCET and HQET plus the effective heavy-to-light current, which was also obtained including $O\left(\lambda^{2}\right)$ corrections. Aspects of the $O(\lambda)$ corrections were previously investigated in [7].

Among the 2 (5) leading-order symmetry relations between the transition form factors for $B$ to pseudoscalar (vector) mesons, 1 (3) receive power corrections at order $\sqrt{\Lambda_{\mathrm{QCD}} / m_{b}}$. Whether these are further suppressed dynamically remains to be investigated. To accuracy $\Lambda_{\mathrm{QCD}} / m_{b}$ no form factor relations are left. Phenomenological analyses of the corresponding decays thus remain dependent on models for the relevant hadronic physics.

\section{REFERENCES}

1. M. Beneke, G. Buchalla, M. Neubert and C. T. Sachrajda, Nucl. Phys. B 591 (2000) 313 hep-ph/0006124.

2. J. Charles, A. Le Yaouanc, L. Oliver, O. Pène and J. C. Raynal, Phys. Rev. D 60 (1999) 014001 hep-ph/9812358].

3. M. Beneke, A. P. Chapovsky, M. Diehl and Th. Feldmann, hep-ph/0206152, Nucl. Phys. $\mathrm{B}$ (in press).

4. M. Beneke and Th. Feldmann, Nucl. Phys. B 592 (2001) 3 hep-ph/0008255.

5. C. W. Bauer, S. Fleming and M. E. Luke, Phys. Rev. D 63 (2001) 014006 hep$\mathrm{ph} / 0005275$; 
C. W. Bauer, S. Fleming, D. Pirjol and I. W. Stewart, Phys. Rev. D 63 (2001) 114020 hep-ph/0011336;

C. W. Bauer, D. Pirjol and I. W. Stewart, Phys. Rev. D 65 (2002) 054022 hep$\mathrm{ph} / 0109045$.

6. G. Burdman, Phys. Rev. D 57 (1998) 4254 hep-ph/9710550.

7. J. Chay and C. Kim, Phys. Rev. D 65 (2002) 114016 hep-ph/0201197;

C. W. Bauer, D. Pirjol and I. W. Stewart, hep-ph/0205289. 